VIII ${ }^{\text {èmes }}$ Journées Nationales Génie Civil - Génie Côtier, Compiègne, 7-9 septembre 2004

\title{
Amélioration de la stabilité des supports d'éolienne offshore : mesure du couple de renversement sur des embases gravitaires.
}

\author{
Guillaume Carpentier ${ }^{(a)}$, Michel Bélorgey ${ }^{(b)}$, \\ Christophe Colmard ${ }^{(\mathrm{c})}$, Yves Stassen ${ }^{(\mathrm{d})}$
}

(a)Doctorant, M2C-Groupe Mécanique des Fluides UMR-CNRS 6143

Université de Caen

(b)Professeur, M2C-Groupe Mécanique des Fluides UMR-CNRS 6143

(c)Ingénieur, SAIPEM S.A. 78884 Saint Quentin en Yvelines

(d)Ingénieur, SAIPEM S.A 78884 Saint Quentin en Yvelines

\section{Résumé}

L’implantation d'éoliennes en mer nécessite de connaître les conditions de stabilité des supports de ces structures face aux forces exercées par la houle. Les résultats que nous présentons ici portent sur l'analyse de la condition de non basculement d'une structure support, de concept Seaflower (SAIPEM) avec caissons gravitaires. Or les formules classiques définies pour des cylindres horizontaux de section uniforme, ne conviennent pas à ce type de structures constituées d'éléments cylindriques verticaux. Dans notre analyse nous avons dissocié les efforts exercés par la houle sur différents types d'embases gravitaires à ceux exercés sur la structure complète. Nos premiers résultats ont permis de proposer des solutions d'optimisation des embases gravitaires et de quantifier l'influence du mât.

\section{$\underline{\text { Abstract }}$}

Installation of offshore windmill requires to understand stability conditions against wave load. The formulation of Morison is traditionally used to described the total load for fixed cylinder (Hald 2002). However this relation is recognized as not fit well to the study of vertical cylinder almost if this one is made up. In the analysis of the stability we studied the inversion of structures according to the Seaflower concept (Saipem) with gravity boxes. These first results to the measure of the torque reaction, enabled us to optimize the concept and to give a solution more stable for that kind of gravity structure.

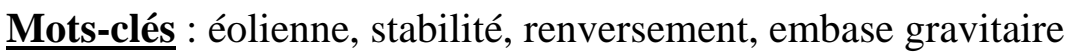

\section{Introduction}

Dans le contexte des accords de Kyoto, les gouvernements occidentaux se tournent naturellement vers les énergies renouvelables. Actuellement, l'énergie éolienne s'impose comme une des plus rentables, particulièrement en milieu marin où les vents sont relativement réguliers. Il existe aujourd'hui plusieurs types d'implantations :

- Les mono-pieux, sur fonds sableux. (Danemark), mais ils se sont avérés peu stables. En effet les vibrations générées par la houle tendent à enfoncer la structure.

- Les 'suction caissons' adaptés aux fonds vaseux, mais ils ne sont pas encore complètement stables malgré les investigations Anglaise et Australienne.

- Les caisson poids tel que développé dans le projet Seaflower, relativement stables, facilement implantables sur bon nombre de sites. Ils semblent une solution plus adaptée au développement des fermes éoliennes.

L’étude de la stabilité de ces structures nécessite l'analyse des efforts exercés par la houle sur l'ouvrage et en particulier :

- La composante horizontale pour la condition de glissement. 
- Le couple de renversement pour la condition de basculement

- Les sous pressions qui réduisent la composante gravitaire et influent sur les deux précédentes conditions.

Ces considérations nous ont conduit à aborder l'étude avec pour objectif, l'optimisation du design de la structure permettant d'aboutir à la réduction des efforts horizontaux sans modification de la composante gravitaire.

Les résultats que nous présentons ici portent sur l'analyse du couple de renversement pour deux types d'embase gravitaire.

\section{Dispositif expérimental et instrumentation}

\subsection{Description des embases}

Le choix de nos modèles, comme celui de nos conditions expérimentales, furent basés sur les résultats du projet COEF (Catalogue Offshore Eolien Français) défini entre l'ADEME (Agence de l'Environnement et de la Maîtrise de l'Energie) et Bouygues Offshore. (Figure 1 \& Tableau)

Tableau 1 : Description des éoliennes et de leurs sites d'implantation

\begin{tabular}{|c|c|c|c|c|c|c|}
\hline \multicolumn{4}{|c|}{ Projet COEF } & \multicolumn{3}{c|}{ Projet SEAFLOWER } \\
\hline \multicolumn{3}{|c|}{ Houle } & \multicolumn{2}{c|}{ Caisson } & Mât \\
\hline Profondeur(m) & Localisation & Hmax (m) & T (s) & $\begin{array}{c}\text { Diamètre } \\
(\mathrm{m})\end{array}$ & $\begin{array}{c}\text { Hauteur } \\
(\mathrm{m})\end{array}$ & $\begin{array}{c}\text { Diamètre } \\
(\mathrm{m})\end{array}$ \\
\hline 15 & $\begin{array}{c}\text { Mer du } \\
\text { nord }\end{array}$ & 8 & 10 & 30 & 11 & 5 \\
\hline 25 & Cotentin & 13 & 13 & 24 & 11 & 5 \\
\hline 35 & Bretagne & 13 & 13 & 34 & 15 & 5 \\
\hline 50 & Languedoc & 12 & 12 & 34 & 12 & 5 \\
\hline
\end{tabular}
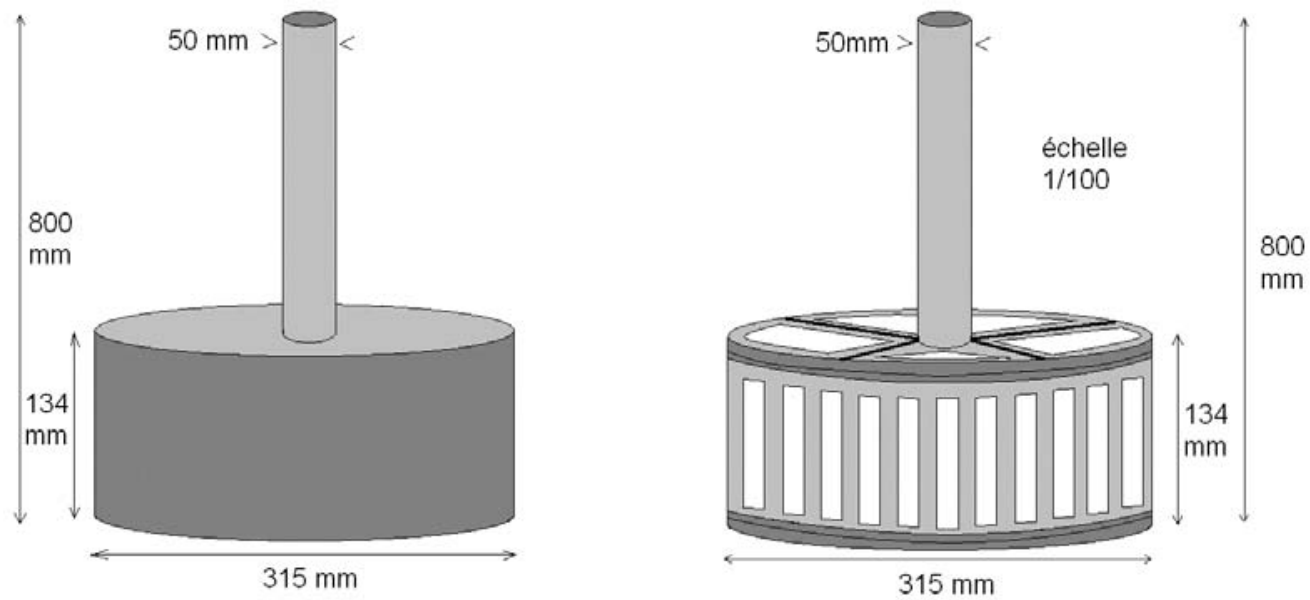

Figure 1: Design des embases étudiées

Dans l'idée de valider un maximum de configurations nous avons pris une échelle de $1 / 100$, ce qui nous permets de négliger la diffraction pour la tête du mât, c'est-à-dire un rapport : diamètre du mât/largeur du canal $<10$ la où les vitesses de la houle sont les plus importantes. Quant au caisson, nous supposons que les vitesses sur le fond sont suffisamment faibles pour négliger la diffraction. 


\subsection{Dispositif expérimental}

Les études ont été réalisées dans le canal à houle de 22 mètres de long pour 0,8 mètre de large du groupe Mécanique des Fluides de l'UMR-CNRS 6143 (M2C). La hauteur d'eau utile était de 0,5 mètre, pour nous placer dans les conditions les plus hostiles. Dans la même idée les amplitudes de houle choisies étaient comprises entre 25 et $100 \mathrm{~mm}$ (soit 2,5 à 10 mètres en conditions réelles), pour des périodes de 0,7 à 2,5 secondes ( 7 à 25 secondes en conditions réelles).

Le canal est muni d'un générateur de houle de type absorbeur et comporte à son extrémité une plage d'amortissement en mousse de nid d'abeille. L'ensemble de ce dispositif nous permet de réduire la réflexion de la houle à une valeur inférieure à 7\%.

\subsection{Instrumentation}

Les caractéristiques de la houle $(\mathrm{H}, \mathrm{L}, \mathrm{T})$ ont été déterminées à l'aide de triplets de limnimètres (sondes résistives classiques), par application de la méthode de Mansard et Funke. Nous avons disposé deux triplets de sondes, un avant la structure pour la mesure des houles incidentes et réfléchies, un autre à l'aval pour la mesure de la houle transmise.

La détermination du Run-up a été quantifiée à l'aide d'une sonde résistive placée sur la génératrice amont du mât, associée à une autre située dans la même section droite du canal, mais éloignée du mât.

Les efforts exercés par la houle sur la structure (et en particulier le couple de renversement) ont été déterminés à l'aide de 4 taquets éprouvette, en duralumin, munis de jauges de contraintes Vishay de type CEA. Nous avons choisi cette technique car elle correspondait plus à la nature des sollicitations sol/structure subies par l'ouvrage. En effet, le fait de maintenir l'ouvrage par les fondations nous rapproche des conditions de fonctionnement réel. Les résultats que nous présentons ici correspondent à la première approche dans le cas de soubassement fixe et imperméable (exemple des roches). L'étude sera poursuivie avec des fonds perméables.

Les 4 taquets étaient fixés sur la maquette qui, par leur intermédiaire, reposait ainsi sur le sol par 4 pieds (figure 2). Leur disposition était la suivante :

- Deux dans le plan médian de propagation de la houle qui permettent de quantifier les action de renversement et deux dans le plan perpendiculaire qui mesurent les efforts transverses (exemple lâcher de tourbillons pour les grands nombres de Strouhal).

- Un ( $\left.{ }^{\circ} 1\right)$ au droit de la génératrice amont de l’embase gravitaire.

- Un (n³) au droit de la génératrice aval de la structure gravitaire.

- Les deux autres $\left(n^{\circ} 2\right.$ et 4$)$ au droit des génératrices de la structure gravitaire, situées dans la section droite perpendiculaire à l'axe du canal.

Après étalonnage et remise à zéro des valeurs pour la maquette en position sans houle, la mesure des contraintes sur les 4 taquets nous permettait de déterminer les actions de la houle sur la structure. En particulier, les taquets $\mathrm{n}^{\circ} 1$ et 3 nous ont permis de mesurer le couple de renversement. La figure 3 donne un exemple d'enregistrement des efforts sur chacun des taquets. 

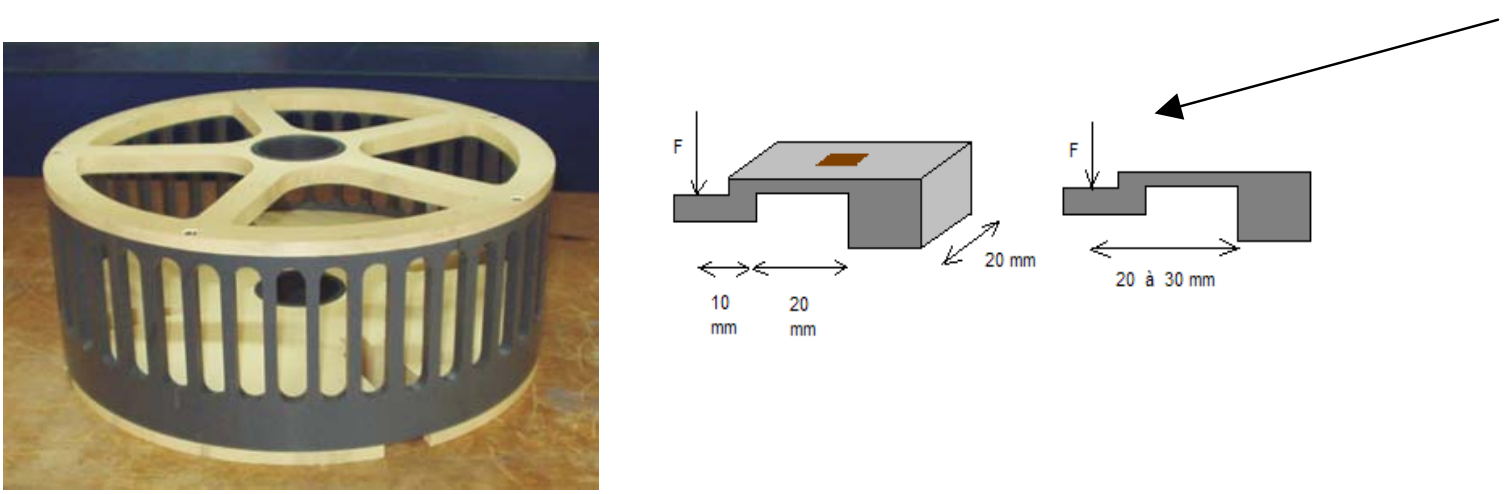

Figure 2: Description et position des capteurs

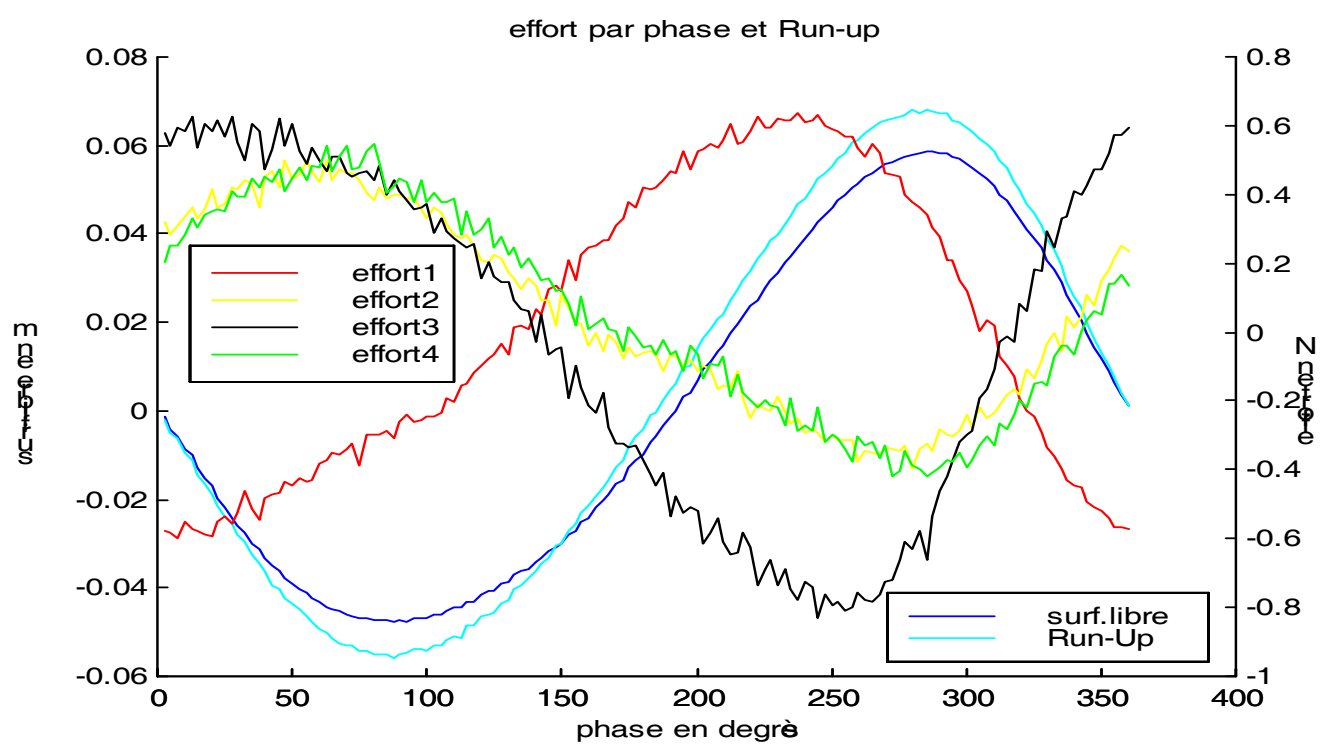

Figure 3: Exemple d'enregistrement des efforts enregistrés sur chaque taquet, pour une houle $H=100 \mathrm{~mm} ; T=1.78 \mathrm{~s}$

\subsection{Traitement de l'acquisition}

Lors de cette campagne de mesures, nous avons réalisé une analyse temporelle et statistique à partir d'un découpage en 'Zero-Up Crossing'. Pour accentuer la précision de cette méthode nous avons allongé notre temps d'acquisition (180 s). Afin d'en améliorer la validité nous avons aussi augmenter la fréquence d'acquisition $(128 \mathrm{~Hz})$. De cette manière nous avons pu augmenter le nombre de phases (144) lors du découpage par rapport à notre vague moyenne afin d'obtenir plus de point de mesure (60) par phase.

\section{$\underline{\text { 3.Analyse de la stabilité }}$}

La maquette est fixée en quatre points sous l'embase gravitaire. La mesure des efforts en chacun des points nous permet de déterminer, en fonction de la phase de la houle, les éléments suivants:

- La composante verticale des efforts.

- Le couple de renversement. 
Pour notre étude et pour cette première approche, nous avons formulé l'hypothèse que la composante verticale de l'action de la houle se répartissait uniformément sur les 4 capteurs. Les capteurs latéraux 2 et 4 nous permettaient alors d'en déterminer l'intensité.

Compte tenu de cette information, les capteurs 1 et 3 permettaient alors de déterminer le couple de renversement associé à la composante horizontale de l'action de la houle. Nous avons pour ce cas considéré le moment de l'effort au point correspondant au centre du pied de mât.

En ce qui concerne la composante verticale des actions de la houle, les enregistrements des capteurs 2 et 4 montrent une évolution sinusoïdale en fonction de la phase de la houle. C'est-à-dire des efforts verticaux présentant des maximums et des minimums. Cette évolution en fonction de la phase de la houle correspond à la combinaison de deux phénomènes :

- L'évolution des surpressions dues au passage de la houle sur la partie supérieure de l'embase gravitaire.

- L'évolution des sous-pressions sous le caisson

Les maximums correspondent à ce que nous avons appelé les phases d'appuis, car ils renforcent la stabilité de l'ouvrage.

Les minimums correspondent à des phases de la houle pour lesquelles les sous-pressions sous l'embase gravitaire sont maximales, réduisant ainsi la condition de stabilité par glissement. Nous les avons appelé phase de portance.

\subsection{Embase gravitaire pleine seule}

Nous avons analysé le couple de renversement et les efforts d'appui et de portance en fonction des caractéristiques de la houle. Sur la figure 4 nous avons tracé l'évolution du couple de renversement en fonction de la cambrure $\mathrm{H} / \mathrm{L}$ de la houle et sur la figure 5, l'évolution des forces d'appui et de portance.

Nos résultats mettent en évidence :

- Une évolution linéaire du couple de renversement en fonction de la cambrure.

- Un effet de portance plus important que l'effet d'appui pour les fortes cambrures.

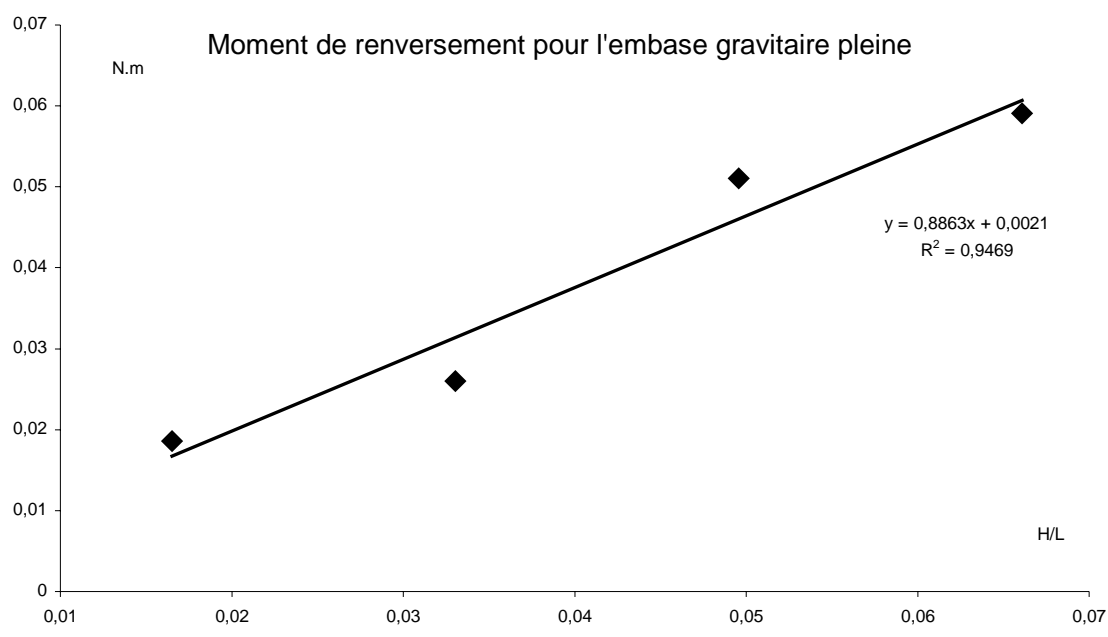

Figure 4: Evolution du couple de renversement sur l'embase pleine en fonction de la cambrure de la houle. 


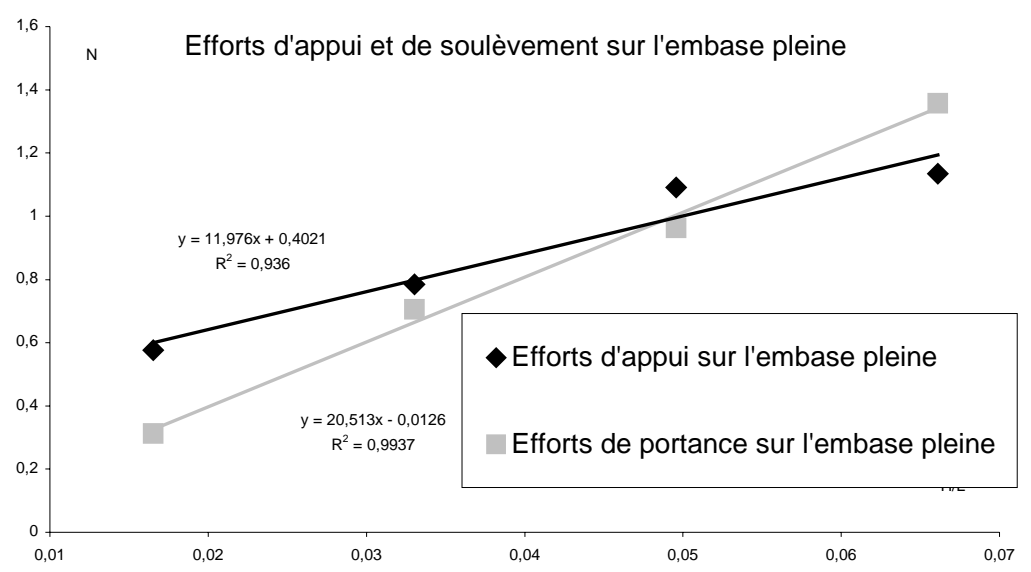

Figure 5: Evolution des effets d'appui et de portance sur l'embase pleine en fonction de la cambrure de la houle.

\subsection{Embase gravitaire perforée seule}

Ici aussi, nous avons analysé le couple de renversement et les efforts d'appui et de portance en fonction des caractéristiques de la houle. Sur la figure 6 nous avons tracé l'évolution du couple de renversement en fonction de la cambrure $\mathrm{H} / \mathrm{L}$ de la houle et sur la figure 7, l'évolution des forces d'appui et de portance.

Nos résultats mettent en évidence :

- Une évolution linéaire du couple de renversement en fonction de la cambrure, comme dans le cas précédent.

- Un effet de portance qui reste moins important que l'effet d'appui quelque soit la cambrure.

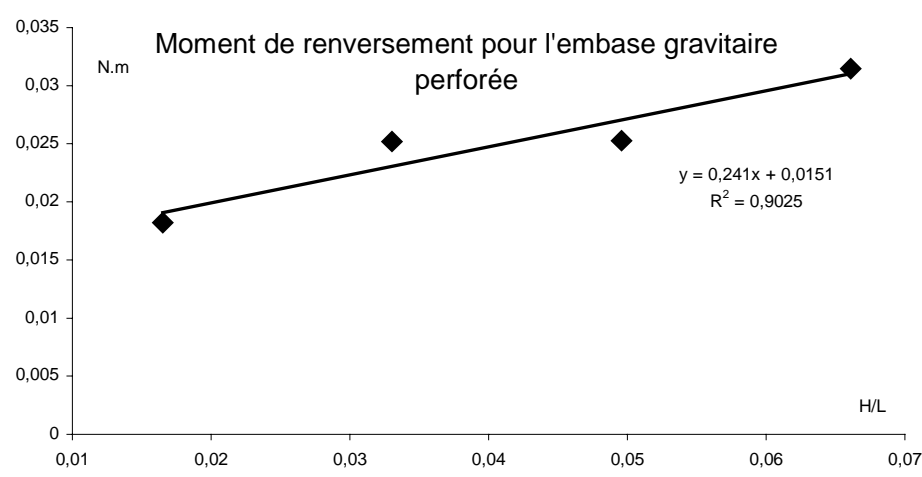

Figure 6: Evolution du couple de renversement sur l'embase perforée en fonction de la cambrure de la houle. 


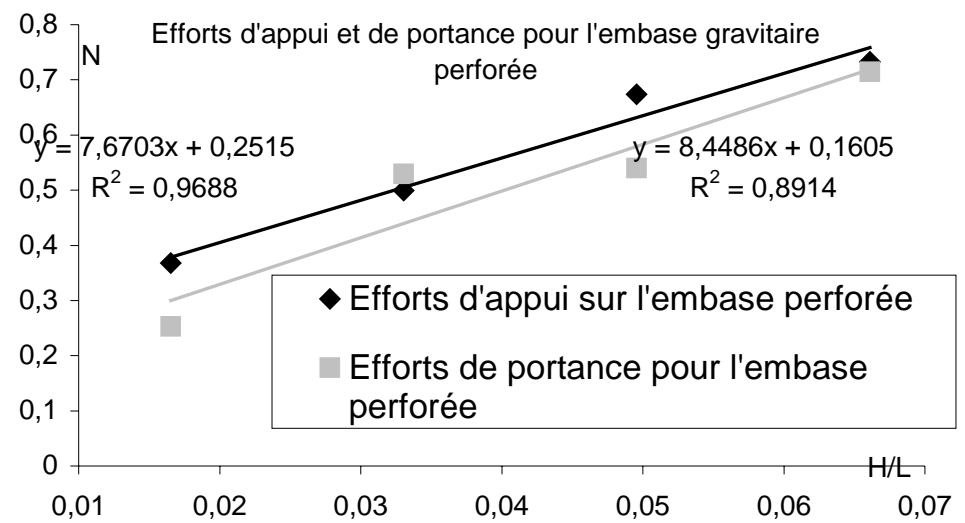

Figure 7: Evolution des effets d'appui et de portance sur l'embase perforée en fonction de la cambrure de la houle.

Par ailleurs, une étude par phase nous a montré que les efforts de portance et d'appui étaient en opposition de phase par rapport à ceux mesuré avec le caisson plein .Cela nous permet de penser qu'en jouant sur le déphasage des efforts via la porosité nous pourrons encore accroître la stabilisé ces structures.

\subsection{Structures complètes avec le mât}

Ici aussi, sur les structures complètes avec mât, nous avons analysé le couple de renversement et les efforts d'appui et de portance en fonction des caractéristiques de la houle. Sur la figure 8a nous avons tracé l'évolution des couples de renversement en fonction de la cambrure $\mathrm{H} / \mathrm{L}$ de la houle et sur la figure 8b, l'évolution des forces d'appui et de portance.

Nos résultats mettent en évidence l'importance de l'influence du mât sur le couple de renversement. En effet, si la variation reste linéaire en fonction de la cambrure, les résultats indiquent, dans l'un et l'autre cas, une intensité beaucoup plus importante du couple de renversement avec la présence du mât. Le facteur d'augmentation plus important dans le cas de la structure pleine que dans le cas de la structure perforée nous autorise à penser que cette différence est due, entre autres, à l'influence du Run-up qui est différent dans l'un et l'autre cas.

Par contre, en ce qui concerne les forces d'appui et de portance, si l'évolution présente des écarts relatifs importants dans le cas de la structure perforée, leur intensité reste sensiblement du même ordre de grandeur que sur les embases seules. 


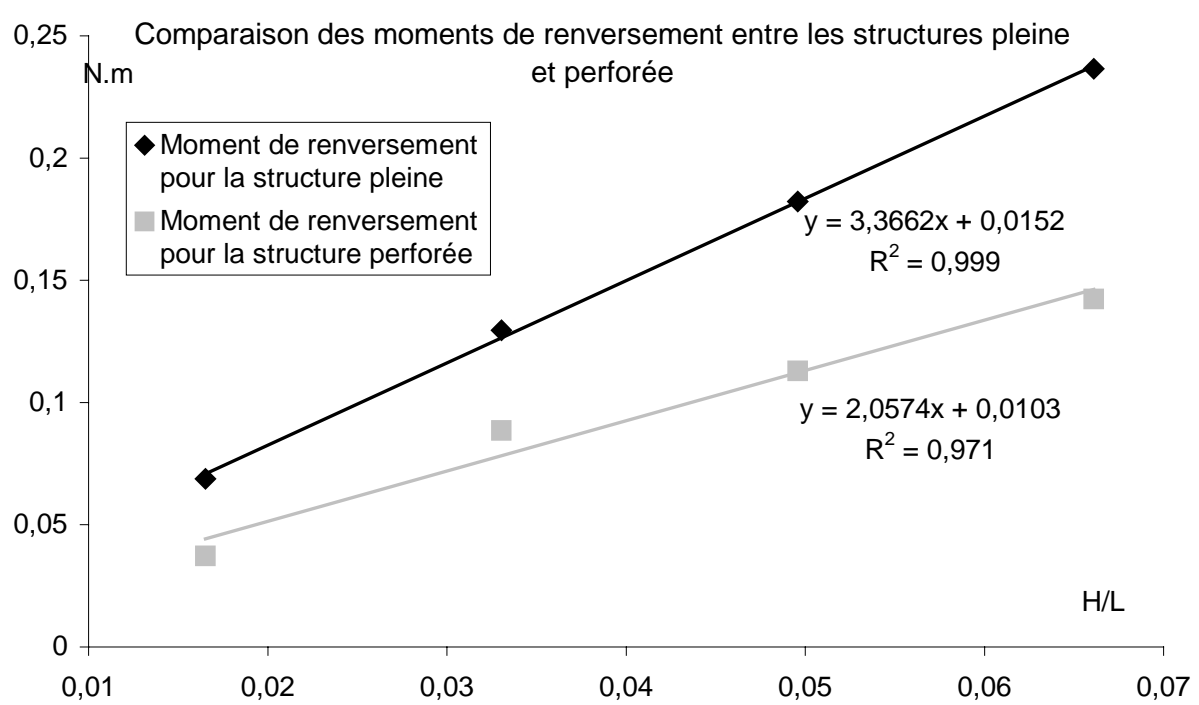

Figure 8a: Evolution du couple des efforts associés au renversement en fonction de la cambrure pour les caissons gravitaires plein et perforé munis du mât.

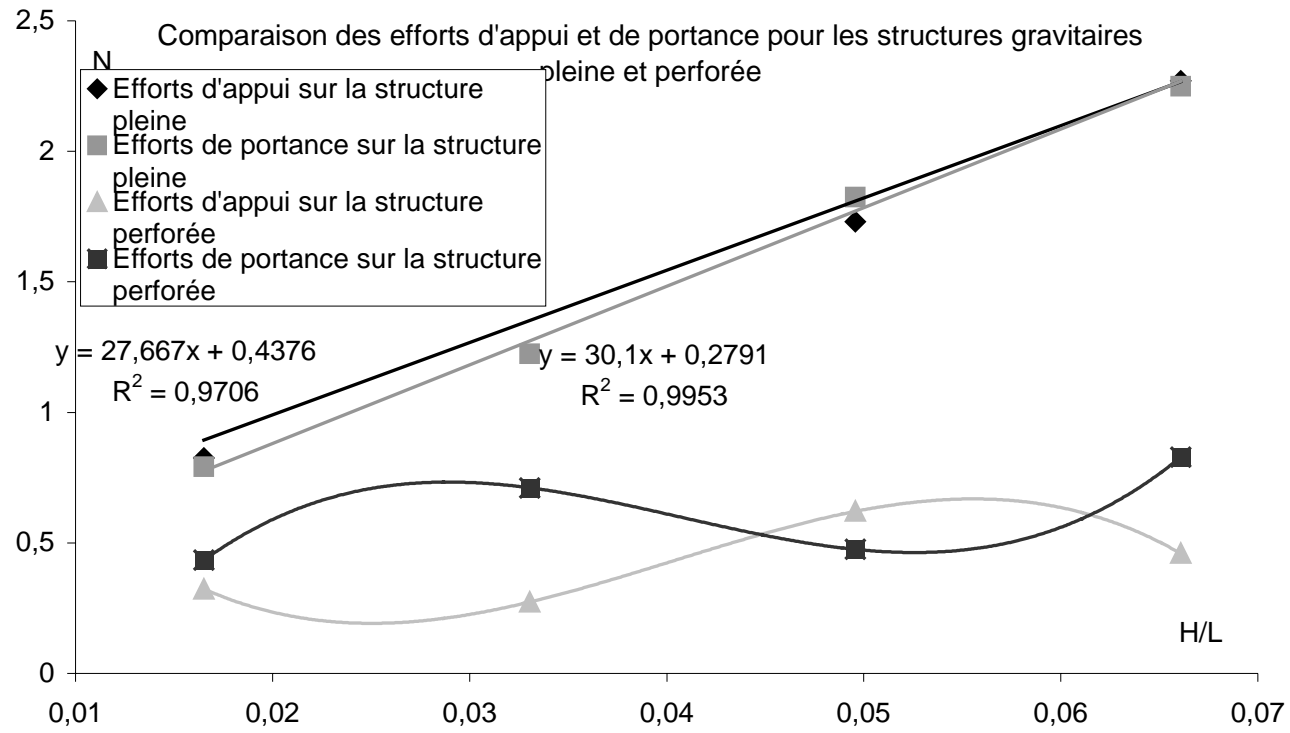

Figures 8b: Evolution des efforts associés à l'appui et à la portance en fonction de la cambrure pour les caissons gravitaire plein et perforé munis du mât.

Par ailleurs, à la lecture de ces différents graphiques, on remarque que le caisson perforé avec ou sans mât est deux fois moins sensible au renversement quelque soit la cambrure de la houle. 


\subsection{Etude du Run-up}

A l’aide de la sonde résistive placée sur la génératrice amont du mât, il nous a été possible de mesurer le Run-up sur chacune des structures. La figure 9 présente les résultats en fonction de la cambrure de la houle.
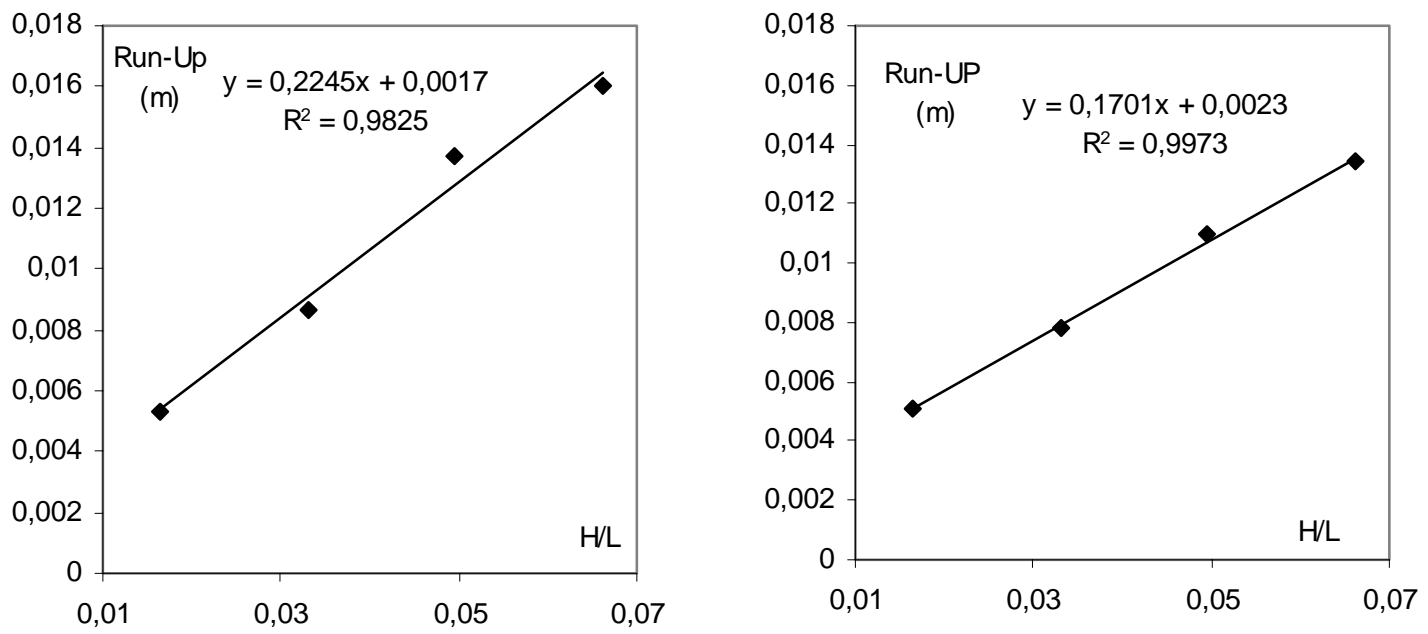

Figure 9: Evolution du Run-up en fonction de la cambrure pour les deux structures complètes (à gauche avec le caisson plein ; à droite avec le caisson perforé)

Nos résultats mettent en évidence un Run-up moins important dans le cas de la structure perforée. On peut attribuer cette différence à la nature différente de l'écoulement et de la turbulence au voisinage des embases. La structure perforée étant plus «transparente » à l'écoulement. Cette différence sur l'intensité du Run-up peut expliquer en partie la différence entre les couples de renversement sur la structure perforée et la structure pleine.

\section{Conclusion}

Si notre hypothèse de répartition, sur les quatre pieds de la structure, de l'efforts associé à la composante verticale de l'action de la houle demande à être confirmée, notre étude nous a permis de quantifier le couple de renversement de caissons gravitaires support d'éolienne côtière. Nous avons mis en évidence l'importance de la nature «transparente » de la structure tant sur le couple de renversement que sur l'importance du Run-up au niveau du mât.

Les caissons perforés apportent une solution qui limite fortement ce renversement. Mais l'optimisation de telles structures nécessite d'analyser finement la nature de l'écoulement, (champ moyen et champ turbulent) au voisinage de l'embase et au niveau de l'implantation du mât (raccord entre le mât et l'embase). C'est pourquoi l'étude sera poursuivie en tenant compte du niveau de drainage des soubassements.

Pour affiner le dimensionnement de tel ouvrage il est également essentiel de bien séparer les efforts horizontaux et verticaux et d'analyser l'évolution des sous-pressions sous la structure. 


\section{Références}

1.Duperron D., (2002). Etude et optimisation d'une solution d'embase gravitaire pour éolienne offshore. Rapport Saipem

2.Hald T., (2002). Wave induced loading on wind mill foundations in Danish waters. ICCE 2002.

3.Mansard et Funke, (1980). The measurement of Incident and reflected Spectra using a least square Method. $17^{\text {th }}$ Coastal Engineering conference, Sydney, Australia.

4.CETMEF, (Juin 2002).Conditions de glissement sous les ouvrages poids portuaires. Etude bibliographique.

5.Capentier G., Bélorgey M. (2003). Stabilité des caissons isolés. Rapports 1 \& 2 Saipem. 